\title{
Vision base Tool Monitoring System for a Reconfigure Micro Factory System
}

\author{
Mohit Pant \\ Astt. Prof. \\ Shri Siddhi Vinayak Group of \\ Institute, Bareilly
}

\author{
Sandeep Sunori \\ Astt. Prof. \\ Graphic Era Hill University, \\ Bhimtal Campus
}

\author{
Anu Gupta \\ Teaching Associate \\ Shri Siddhi Vinayak Group of \\ institute, Bareilly
}

\begin{abstract}
During the past decade, new sensing technologies, such as are optical measurement, image processing (edge detection, pattern matching, $\mathrm{x}$-ray image processing), acceleration sensor, vibration sensor, inductive loops, laser range scanners, computer vision sensors have been greatly enhanced and applied to the Intelligent in Automatic Tool Wear Monitoring System (ATWMS) area. On-line tool monitoring system may realize substantial cost saving through image processing. Currently, tool wear monitoring performed by vibration, inductive and debris sensors. Oil debris sensor is a popular measurement device used to collect oil condition data. This sensor generates an output signature with the passage of a metallic particle through the oil return lines. However, the signal measured through the oil debris sensor is severely tainted by various noises, e.g., the background noise present as well as the interferences caused by the vibrations of the structure where the sensor is mounted. These interferences affect the performance of the health assessment unit considerably. As such this paper focuses on the tool condition monitoring direct by metal chips using image processing technology.
\end{abstract}

\section{Keywords}

Breakage function, Debris analysis, tool monitoring, thresholding, filtering and metal chips.

\section{INTRODUCTION}

Principally tool wear monitoring system can be classified into two groups, where if they measure tool wear directly at the cutting edge of the worn tool (direct measurement techniques) or if the measured parameter or a signal of cutting process allow to dray conclusion upon the degree of tool wear (indirect measurement techniques).Indirect measurement technique allows to monitor cutting process on-line, which is one of their biggest advantages. Example for direct measurement techniques are optical measurement using tool markers microscope or tactile sensors. Among indirect measurement techniques the analysis of acoustic emission signals, the measurement of cutting force or spindle torque have become established monitoring techniques. Vibration, acoustic emission and inductive loop sensors are examples of the indirect measurement techniques.

Although the amount of wear information provided by the tactile sensing technique has found way into practice due to its robustness, it is different with optical optoelectronic measuring principal, where automation of measurement process is difficult.
In most cases indirect measuring techniques are employed for atomize tool condition monitoring. These are based upon analysis of typical signals of cutting process. Normally, these tool wear monitoring system are based upon the comparison of a reference signal of an optimized cutting process with the actual process signal. Once actual signal has changed beyond a threshold value, an alarm is given in order to stop the cutting process and indicate a request for tool change. As consequence, these type of systems only useful for monitoring the production of large batches, where identical cutting operation are repeated many times. Indirect tool wear monitoring technique are rather complicated to deal with and therefore in convenient to handling. In practice obtaining suitable reference signal from an undisturbed cutting process requires lot of efforts and skills. Once the proper reference signal has been obtained, another problem arises when it comes to industrial practice [1].

The term Metal chips Particle or metal chips Debris relates from the field of "Tribology". Tribology is the study of tool wear condition by analysis of metal chips [2]. Small metal particles (chips) that generated after machining are produced in all machines containing moving parts in contact. After the initial run period of any machine which usually produces large amount of chips debris due to the friction between tool and machining object, the operation of the machine reaches a steady state and then produces normal amount of chips debris. Any change in the steady state operation of the machine creates a change in the normal wear mechanism. This change will generate small change in metals chip size that produce by machining. This change will generate small change in metals chip size that produced by machining. Tool wear debris transported through oil lubricant system [3].

These systems provide important information about steady state operation. Coolant condition data is a major source of information for tool condition monitoring in Debris analysis. It contains information about the metallic particle content and thus reference level of wear and fatigue induce damage in mechanical tool. Analysis of the measured data leads to an estimate of the size and number of metallic particles present in the lubricating oil and consequently health state of the tool wear [3,4]. These wear mechanisms give plate particles a rough surface and an irregular perimeter. Small particles often develop in connection with roller bearings.

There are six basic particles type generated through the wear process.

a) Normal Rubbing Wear: Rubbing wear particles are generated because of normal sliding wear in a machine and result from exploitation of particles of the shear mixed layer [5]. 
b) Cutting Wear Particles: Cutting wear particles are generated as result of one surface penetrating another. Cutting wear particles are abnormal. Their presence and quantity should be carefully monitored. If the majority of the cutting particles in a system are a few micrometers long and a fraction of micrometers wide the presence of particulate contaminants should be suspected [5].

c) Spherical Particles: These particles are generated in the bearing cracks. If generates their presence gives an improved warning of impending trouble as they are detectable before any sapling occurs [5].

d) Severe Sliding: Severe sliding wear particles are identified by parallel on their surfaces. They are generally larger than 15 microns, with the length-towidth thickness ratio falling between 5-30 microns [5].

e) Bearing Wear Particles: These distinct particle types have been associated with rolling bearing fatigues. These
Particles reach a maximum size of 100 microns during the micros palling process. They range between 20 to 50 microns in major diameter with a thickness ratio of $30: 1$ [5].

f) Gear Wear: Two types of wear have been associated with gear wear. They generally have a smooth surface and frequently irregularly shaped. Depending upon the gear design, the particles usually have a major dimension-to thickness ration between $4: 1$ and 10:1. Scuffing or scoring particles are caused by too high a load and / or speed. These particles tend to have a rough surface and jagged circumference. Even small particles may be discerned from rubbing wear by these characteristics [5].

\subsection{Wear process monitoring technique}

The method of wear process can be classified into three main types, which are shown in figure.

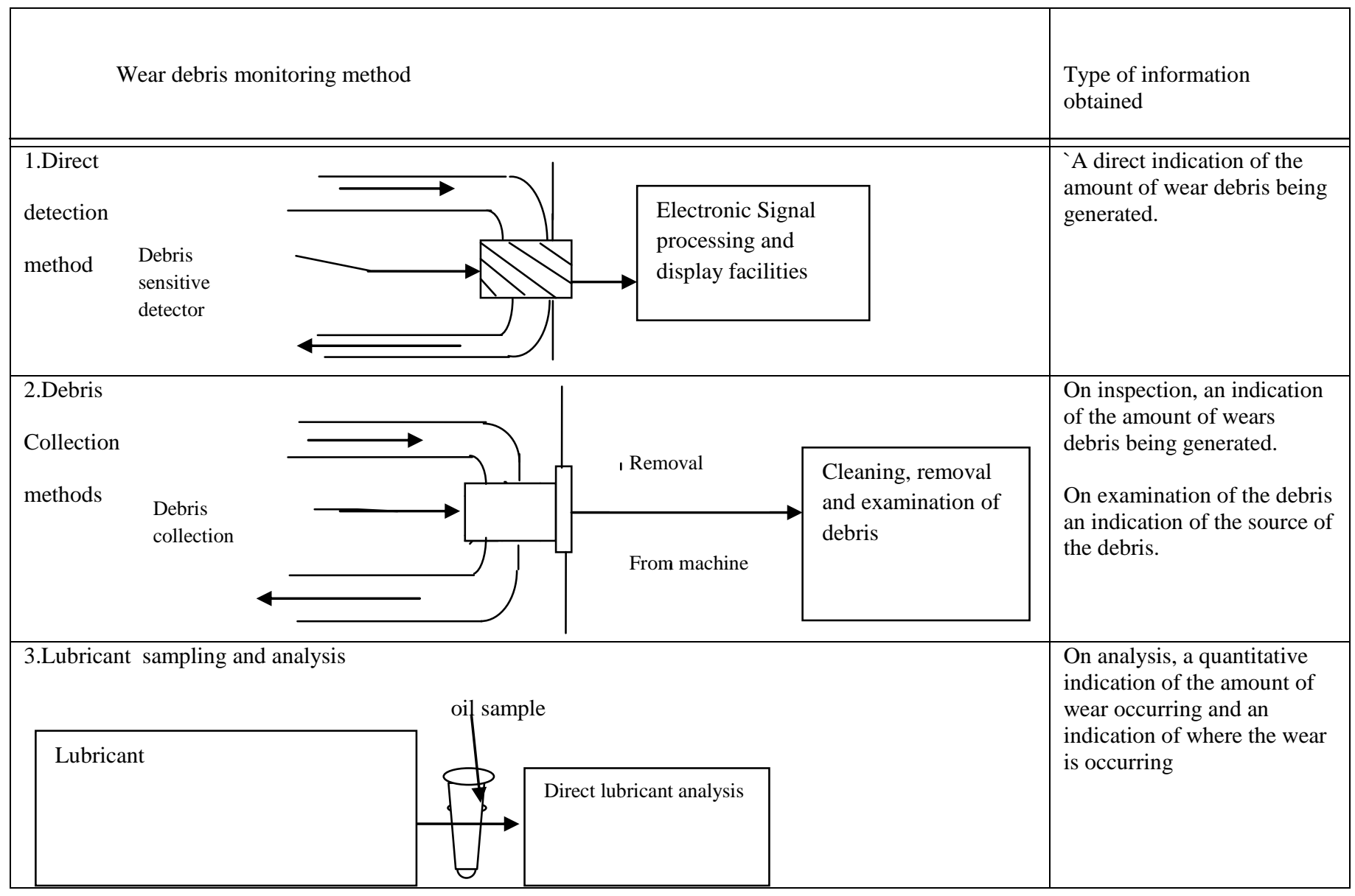

Figure 1: Wear process monitoring technique

a) Direct detection method: Wear debris in the lubricant is detected in the machine by arranging for the oil flow through a device, which is sensitive to the presence of debris $[6,7]$.

b) Debris collection methods: Wear debris is collected in a device, fitted to the machine which is convenient to remove, so that the debris can be extracted for examination [7].

c) Lubricant Sample Analysis: A sample of lubricant is extracted from the machine and analyzed for wear debris contamination. These methods are normally used to monitor the conditions of components lubricated by a circulatory oil system [7]. 


\subsection{Disadvantage of Debris analysis}

When applying a wear debris monitoring method to any machine for the first time there is an initial learning period required, partly to gain experience in using the equipment, but mainly to establish wear debris characteristic levels which indicate normal and incipient failure conditions. This learning period can take up to 2 Yrs. During this time it will also be necessary to establish the inspection and sampling intervals for intermittent monitoring methods such as debris collection and lubricant sampling. This time interval will depend on the application but fortnightly or monthly is probably a reasonable choice for an industrial application in the absence of more precise guidance.

In our experiential setup we take sample of oil from return line on a small piece of paper. When oil comes to connect with paper surface as large amount of oil absorbed by paper surface and thin layer of small metal partial will formed on paper surface. This thin layer used as a sample. After taking sample we take image of sample by high zoom camera. This color image converts into gray image because binary image can be obtained from gray scale image by thresholding operation. A thresholding operation chooses some of the pixels as the foreground pixel that make up the objects of interest and the rest as background pixels [8].

Given the distribution of gray tones in a given image, certain gray tone value can be chosen as threshold values that separate the pixel into group. In the simple case a single pixel value $t$ is chosen. All pixels whose gray tone value are greater than or equal to't' become foreground pixel and all the rest become background. This threshold is called threshold above. There are many variants including threshold below, which makes the pixel values less than or equal to $t$ the foreground, threshold in side which is given a lower threshold and a upper threshold and selected pixel whose values are between the two as foreground, and threshold outside, which is the opposite of threshold inside [9].

Let $\sigma_{\omega}{ }^{2}$ be the weighted seem sum of group variances that is the within-group variance. Let $\sigma_{1}^{2}(\mathrm{t})$ be variance for group with values less than or equal to $t$ and $\sigma_{2}{ }^{2}(t)$ be variance from the group with greater than $t$. Let $\mathrm{q}_{1}(\mathrm{t})$ the probability for the group with value less than or equal to $t$ and $q_{2}(t)$ be probability for group with value greater than $t$. Than the within-group variance $\sigma \omega^{2}$ is defined by

$$
\begin{aligned}
\sigma_{\omega}{ }^{2}(\mathrm{t}) & =\mathrm{q}_{1}(\mathrm{t}) * \sigma_{1}{ }^{2}(\mathrm{t})+\mathrm{q}_{2}(\mathrm{t}) * \sigma_{2}{ }^{2}(\mathrm{t}) \ldots \ldots \\
\mathrm{q}_{1}(\mathrm{t}) & =\sum_{i=1}^{t} p(i) \ldots \ldots \ldots \ldots \ldots \ldots \ldots \ldots \ldots \ldots \ldots \ldots \ldots \ldots \ldots \ldots \ldots \ldots \ldots \ldots \ldots \ldots
\end{aligned}
$$

Let $\mathrm{u}_{1}(\mathrm{t})$ be the mean for the first group and $\mathrm{u}_{2}(\mathrm{t})$ the mean for the second group.

$\mathrm{u}_{1}(\mathrm{t})=\sum_{t=1}^{t} i \frac{p(t)}{q_{1}(t)}$

$\mathrm{u}_{2}(\mathrm{t})=\sum_{i=t+1}^{I} i \frac{p(t)}{q_{2}(t)}$

$\sigma_{1}^{2}(\mathrm{t})=\sum_{i=1}^{t} \frac{\left(i-u_{1}(t)\right)^{2} p(i)}{q_{1}(t)}$

$\sigma_{2}^{2}(\mathrm{t})=\sum_{i=t+1}^{I} \frac{\left(i-u_{2}(t)\right)^{2} p(i)}{q_{2}(t)}$
The best threshold $\mathrm{t}$ can then be determined by a simple sequential search through all possible value of t to locate the threshold $t$ that minimize $\sigma_{\omega}{ }^{2}(t)$. There is relationship between the within group variables $\sigma_{\omega}{ }^{2}(t)$ and total variable $\sigma^{2}$ that does not depend on the threshold total variables is defined.

$\sigma^{2}=\sum_{i=1}^{I}(i-u)^{2} p(i)$

$\mathrm{u}=\sum_{i=1}^{I} i p(i)$

After getting perfect threshold image we have to remove noise, wiener filtering technique is best option in among of filtering technique. The inverting filtering is the restoration technique for de- convolution, i.e., when image is blurred by know low pass filter, it is possible to recover the image by inverse filtering or generalize inverse filtering. However inverse filtering is very sensitive to additive noise. The winer filtering executes on optimal trod between inverse filtering and noise smoothing. The wiener filters linear estimation of original image. The approach is based on a stochastic framework [10]. The orthogonality principal implies that the wiener filter in Fourier domain can be expressed as follow

$\omega\left(f_{1}, f_{2}\right)=\frac{H^{*}\left(f_{1}, f_{2}\right) S_{x x}\left(f_{1}, f_{2}\right)}{\left|H\left(f_{1}, f_{2}\right)\right|^{2} S_{x x}\left(f_{1}, f_{2}\right)+s_{m}\left(f_{1}, f_{2}\right)}$

Where $s_{x x}\left(f_{1}, f_{2}\right), s_{n n}\left(f_{1}, f_{2}\right)$ power spectra of the original image and the additive are respectively noise, and $H\left(f_{1}, f_{2}\right)$ is the blurring filter. It is easy to see that the Wiener filter has two separate parts, an inverse filtering part and a noise smoothing part. It not only performs the de-convolution by inverse filtering (high pass filtering) but also removes the noise with a compression operation (low pass filtering) [11,12, and 13].

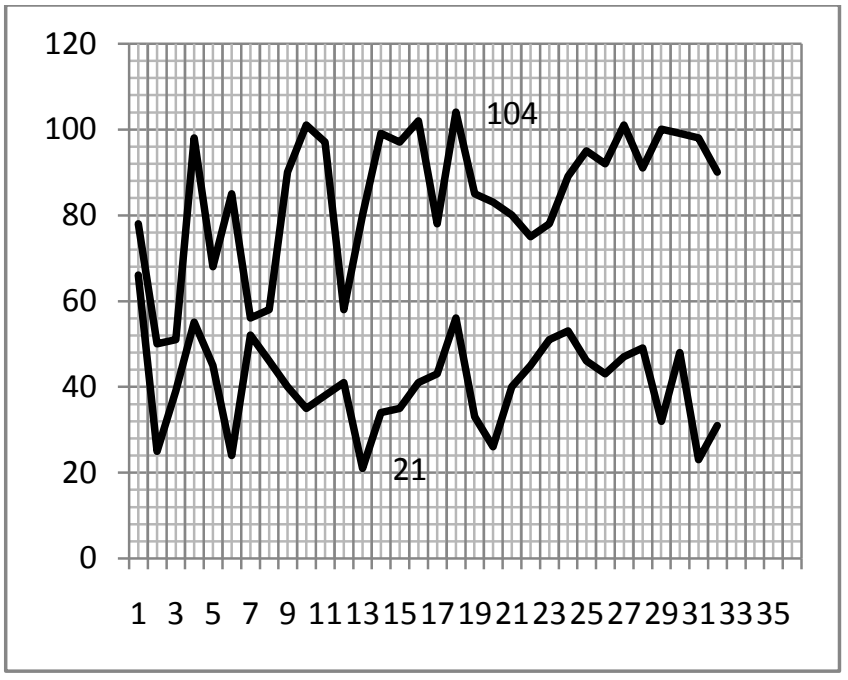

Figure 2: Breakage function

Largest and smallest metal chip will be getting from filtered image. Breakage functions as follow.

$$
a_{1} \leq b(a) \leq a_{2}
$$

Where, $a_{1}=$ area of largest metal chips.

$a_{2}=$ area of smallest metal chips.

$b(a)=$ brakeage function. $a \varepsilon\left(a_{1}, a_{2}\right)$

Brakeage function is dependent upon the material or density of tool and object. At the time of learning system have to take 
30-35 samples to calculate breakage function. If given condition is failed then the system will acknowledge to stop the machining process. During performing the operation on the largest and the smallest metal chip areas $\left(a_{1}\right.$ and $a_{2}$ respectively) shown in above figure 2 . The maximum point signifies the maximum breakage point at $\left(a_{1}=104\right)$ and the minimum point of the graph signifies the minimum breakage point at $\left(a_{2}=21\right)$. After operating these points the testing will be down on the different samples of chips, if the tested result lye between the point $a_{1}$ and $a_{2}$ then than the tool is not damage and working properly. In other condition if the area of the both metal chips goes above from point $\mathrm{a}_{1}$ or goes down from point $\mathrm{a}_{2}$ then the tool will go in the breakage condition.

\section{RESULT AND DISCURSION}

The proposed method was tested we tested with result of five testes on $2.33 \mathrm{GHz}$ PC, $3 \mathrm{~GB}$ RAM. The average correct rate of the tool monitoring system is $88.6 \%$. The resolution of image is $640 \times 480$ and $720 \times 480$.From the table 1 , we observed that for Test second and fifth the average correct rate is less. This is because image in test second and test fifth was taken during night time with low light.

\begin{tabular}{|l|l|l|l|l|l|}
\hline \multicolumn{1}{|c|}{ Table 1: Result compilation table } \\
\hline & First & Second & Third & $\begin{array}{l}\text { Fourt } \\
\text { h }\end{array}$ & Fifth \\
\hline $\begin{array}{l}\text { Total } \\
\text { sample }\end{array}$ & 92 & 375 & 92 & 92 & 92 \\
\hline $\begin{array}{l}\text { Tool Un } \\
\text { brake } \\
\text { condition }\end{array}$ & 58 & 73 & 79 & 54 & 29 \\
\hline $\begin{array}{l}\text { Tool } \\
\text { brake } \\
\text { condition }\end{array}$ & 44 & 302 & 13 & 38 & 63 \\
\hline $\begin{array}{l}\text { \% } \\
\text { Correct } \\
\text { rate }\end{array}$ & 95 & 84 & 89 & 91 & 80 \\
\hline $\begin{array}{l}\text { Average } \\
\text { Correct } \\
\text { Rate }\end{array}$ & & 88.6 & & \\
\hline
\end{tabular}

\section{CONCLUSION}

In our research paper a new technique of "vision base tool monitoring system for a reconfigure micro factory system" which takes very less learning time for tool monitoring. Because in the decade, we have various sensing technologies used such as optical measurement, image processing, acceleration sensor, vibration sensor, inductive loops, laser range scanners and computer vision sensor in area of automatic tool wear monitoring system (ATWMS). But now these days, tool wear monitoring performed by vibration, inductive and debris sensors, which are actually very timeconsuming and fall a bad effect on performance of assessment unit. Due to these above generated problems we proposed a tool monitoring system which directly monitoring the tool through the metal chip.

In tool wear processing technique basically work in three main phases: first one is direct detection method (used for detected wear debris exists in lubricant). Second one is debris collection method (used to collect wear debris with the help of a debris sensor) third one is lubricant sample analysis (used to monitor the condition of components lubricated by a circulatory oil system). After applying these three methods we get perfect threshold image and we have to remove noise by "wiener filtering technique". After filtering we get areas illustrate in fig. 2 for finding breakage function of tool. This whole process takes 2 to 3 days with $86 \%$ correct rate of the tool monitoring system, which is very less from the debris monitoring method, because debris method takes learning period, is 2 year.

Vision based tool monitoring system is reviewed along with new possibilities for reducing the learning period of tool monitoring.

\section{ACKNOWLEDGEMENT}

The authors of this paper would like to thankfully acknowledge Mr. Kuldeep Verma for his constant help.

\section{REFERENCES}

[1] Miller, J. and Kitaljevich, D., 2000, In-line oil debris monitor for aircraft engine condition assessment, IEEE Aerospace Conference Proceedings, Big Sky, MT, USA, 6, 49-56.

[2] Z. Peng, "An integrated intelligence system for wear debris analysis," Wear, vol. 252, no. 9-10, 2002, pp. 730743.

[3] G.A. Khuwaja and M.S. Laghari, "Computer vision techniques for wear debris analysis," Int. Journal of Computer Applications \& Technology, vol. 15, no. 1/2/3, 2002, pp. 70-78.

[4] L. G. Shapiro and G. C. Stockman, Computer Vision, Prentice Hall, New Jersey, 2001

[5] Mohammad Shakeel Laghari and Faheem Ahmed, 2009, Wear Particle Profile Analysis.

[6] Jiang Xufeng, Wang Jianbo, Guo Changying and Zhang Yuan, 2011, Study on Aero-engine Wear Fault Diagnosis by Direct Reading Ferrograph .

[7] M.S. Laghari, "Morphological Analysis of Microscopic Wear Particles," Proc. 2nd Int. Conf. Modeling, Simulation, and Applied Optimization ICMSAO'07, Abu Dhabi, UAE, 2007.

[8] Pankaj Bodoni, Bhupendra Singh and Kuldeep Verma, 2011, "Computer Vision Based currency classification system", International Journal of Computer Applications (0975 - 8887) Volume 16- No.4, February 2011.

[9] M. S. Laghari, F. Ahmed and J. Aziz, 2010, Wear Particle Shape and Edge Detail Analysis.

[10] R.M. Carter and Y. Yan, "Measurement of particle shape using digital imaging techniques," Journal of Physics, vol. 15, 2005, pp. 177-182.

[11] A. Khireddine , K. Benmahammed and W. Puech, 2007, Digital image restoration by Wiener filter in 2D case.

[12] Cherniakov M, Sisov VI, Donskoi L. Synthesis of a periodically time-varying digital filter. VISP 2000;147(5):393-9.

[13] Kumar BVKV, Mahalanobis A, Takessian A. Optimal tradeoffcircular harmonic function correlation filter methods providing con-trolled in-plane rotation response. IP 2000;9(6):1025-34 